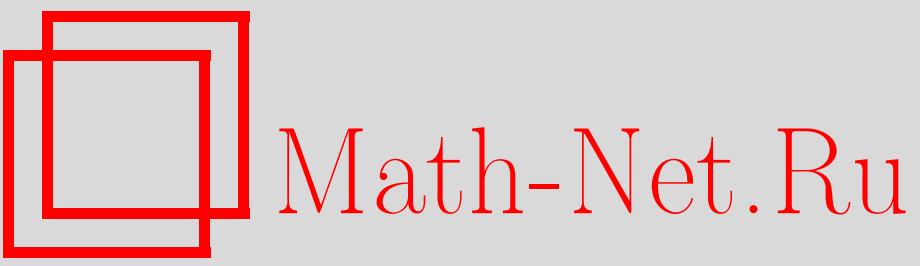

М. М. Глухов, О методах построения систем ортогональных квазигрупп с использованием групп, Матем. вопр. криптогр., 2011, том 2, выпуск 4, 5-24

DOI: https://doi.org/10.4213/mvk40

Использование Общероссийского математического портала Math-Net.Ru подразумевает, что вы прочитали и согласны с пользовательским соглашением

http://www . mathnet.ru/rus/agreement

Параметры загрузки:

IP : 54.198 .55 .26

26 апреля 2023 г., 16:28:01 
УДК 512.548 .7

\title{
О методах построения систем ортогональных квазигрупп с использованием групп
}

\section{Глухов М. М.}

Академия криптографии Российской Федерации, Москва

Получено 23.VI.2010

Предлагается метод построения систем ортогональных квазигрупп и ортогональных латинских квадратов на основе групп Фробениуса. Показано, что в схему этого метода укладываются многие (но не все) известные методы построения ортогональных латинских квадратов с использованием групп.

Ключевые слова: квазигруппа, латинский квадрат, система ортогональных квазигрупп (латинских квадратов), регулярное множество подстановок, регулярный автоморфизм группы

On a method of construction of orthogonal quasigroup systems by means of groups

\section{M. Gluhov}

Academy of Cryptography of Russian Federation, Moscow

\begin{abstract}
We suggest a method of construction of orthogonal quasigroups systems and orthogonal Latin squares by means of Frobenius groups. It is proved that this method generalizes many (but not all) existing methods of orthogonal Latin squares construction by means of groups.
\end{abstract}

Key words: quasigroup, Latin square, system of orthogonal quasigroups, orthogonal Latin squares, regular set of permutations, regular automorphism of a group.

Citation: Mathematical Aspects of Cryptography, 2011, vol. 2, no. 4, pp. 5-24 (Russian). 


\section{§ 1. Предварительные сведения о квазигруппах}

В статье будет использоваться следующая терминология. Подстановка конечного множества $Q$ регулярна, если она разлагается в произведение независимых циклов одной и той же длины. Система (в частности, группа) подстановок множества $Q$ регулярна, если она точно транзитивна, т. е. для любых $a, b \in Q$ содержит ровно одну такую подстановку $g$, что $g(a)=b$, или, иначе, $a g=b$. Отметим, что регулярная группа состоит из регулярных подстановок. Автоморфизм группы $G$ регулярен, если он как подстановка регулярен на множестве всех неединичных элементов группы. Подчеркнем, что регулярный автоморфизм группы $G$ не является регулярной подстановкой на всей группе $G$.

Напомним некоторые понятия из теории квазигрупп и латинских квадратов (см. [1-3], [12-14]).

Квазигруппой называют произвольный группоид $(Q, *)$, в котором при любых $a, b \in Q$ однозначно разрешимо каждое из уравнений

$$
a * x=b, y * a=b .
$$

Квазигруппу с единицей называют лупой. Лупа $(Q, *)$ с ассоциативной операцией * является группой.

В данной работе будут использоваться только конечные квазигруппы. Число элементов квазигруппы называют ее порядком.

Конечную квазигруппу $(Q, *)$ порядка $n$ можно задать таблицей Кэли при любом упорядочении ее элементов во входной строке и входном столбце таблицы. Всюду далее мы будем использовать некоторое одно и то же фиксированное упорядочение элементов из $Q$, которое назовем $\mathrm{cmaндарm-}$ ным. Результат операции $a * b$ будем записывать на пересечении строки таблицы с номером $a$ и столбца с номером $b$.

Из определения квазигруппы следует, что ее таблица является латинским квадратом порядка $n$. И обратно, любой латинский квадрат можно рассматривать как таблицу Кэли квазигруппы при стандартном расположении элементов входной строки и входного столбца. В связи с этим говорят, что квазигруппы являются алгебраической формой задания латинских квадратов.

Каждому элементу $a$ квазигруппы $(Q, *)$ можно сопоставить две подстановки $R_{a}, L_{a}$ на $Q$, определяемые равенствами

$$
x R_{a}=x_{*} a, x L_{a}=a_{*} x .
$$

Они называются соответственно правой и левой трансляциями квазигруппы $(Q, *)$, соответствующими элементу $a$. Множества всех правых и левых трансляций квазигруппы $(Q, *)$ по всем элементам $a \in Q$ обозначим соответственно через $R_{Q}$ и $L_{Q}$. Очевидно, $R_{Q}$ и $L_{Q}$ являются регулярными множест- 
вами. В том случае, когда квазигруппа $(Q, *)$ является группой, $R_{Q}$ и $L_{Q}$ являются соответственно ее правым и левым регулярными представлениями и, значит, являются регулярными группами подстановок.

Легко видеть также, что любое регулярное множество подстановок $H$ из симметрической группы $S(Q)$ совпадает с множеством правых (левых) трансляций подходящей квазигруппы на $Q$. Для построения таблицы Кэли этой квазигруппы достаточно проиндексировать подстановки из $H$ элементами из $Q$ и выписать нижние строчки подстановок $h_{a} \in H$ в столбец в соответствии со стандартным расположением индексов. Операция * в полученной квазигруппе однозначно выражается через подстановки множества $H$ :

$$
a_{*} b=b h_{a} .
$$

Заметим, что латинский квадрат, соответствующий построенной квазигруппе, можно отождествлять с указанным выше упорядоченным множеством подстановок (см., например, [4]).

Квазигруппы $(Q, \circ)$ и $(Q, *)$ называются изотопными, если существуют подстановки $\alpha, \beta, \gamma$ множества $Q$, удовлетворяющие при любых $a, b \in Q$ условию

$$
(a \circ b) \gamma=a \alpha * b \beta .
$$

При этом тройку подстановок $(\alpha, \beta, \gamma)$ называют изотопией квазигруппы $(Q, *)$ на квазигруппу $(Q, \circ)$. Заметим, что таблица Кэли квазигруппы $(Q, \circ)$ получается из таблицы Кэли квазигруппы $(Q, *)$ перестановками строк по подстановке $\beta$, столбцов - по подстановке $\alpha$ и заменой элементов таблицы по подстановке $\gamma^{-1}$. Таким образом, изотопным квазигруппам соответствуют эквивалентные латинские квадраты. В случае, когда $\gamma=\varepsilon-$ тождественная подстановка, изотопию $(\alpha, \beta, \gamma)$ называют главной. При условии $\alpha=\beta=\gamma$ изотопия является изоморфизмом. Легко видеть, что отношение изотопии является отношением эквивалентности на множестве всех квазигрупп, определенных на одном и том же множестве $Q$ и значит, все такие квазигруппы разбиваются на классы изотопных квазигрупп. Эти классы являются укрупнениями классов изоморфных квазигрупп.

Изотопия квазигруппы $(Q, \cdot)$ на себя называется ее автотопией. Все автотопии квазигруппы $(Q, \cdot)$ образуют группу относительно операции умножения отображений. В качестве своей подгруппы она содержит группу всех автоморфизмов $\operatorname{Aut}(Q, \cdot)$.

По квазигруппе $(Q, \cdot)$ можно определить три новых операции $/, \backslash, \times$ на множестве $Q$, положив для любых $a, b, c \in Q$ :

$$
a / b=c \Leftrightarrow c \cdot b=a ; \quad a \backslash b=c \Leftrightarrow a \cdot c=b ; \quad a \times b=c \Leftrightarrow b \cdot a=c .
$$


Операции $/, \backslash, \times$ снова являются квазигрупповыми на множестве $Q$, их (и определяемые ими квазигруппы) называют соответственно левой обратной, правой обратной и сопряженной к основной операции (к квазигруппе $(Q, \cdot))$. Системы квазигрупп, полученные одна из другой заменой всех квазигрупп на сопряженные, условимся также называть сопряженными.

Квазигрупповые операции $\circ$, * и соответствующие им квазигруппы $(Q, \circ),(Q, *)$ называются ортогональными, если при любых $a, b \in Q$ система уравнений

$$
\begin{aligned}
& x \circ y=a, \\
& x * y=b
\end{aligned}
$$

имеет единственное решение относительно неизвестных $x, y$. Отсюда видно, что таблицы Кэли ортогональных квазигрупп при одинаковом расположении элементов во входных строках и столбцах являются ортогональными латинскими квадратами (ОЛК).

Систему различных квазигрупп (латинских квадратов) назовем ортогональной, если в ней ортогональна любая пара различных квазигрупп (латинских квадратов). Для общности систему, состоящую из одной квазигруппы (одного латинского квадрата), будем также называть ортогональной. Ортогональную систему квазигрупп (латинских квадратов) порядка $n$ назовем максимальной, если она не является собственной подсистемой никакой другой ортогональной системы. Очевидно, что ортогональная система квазигрупп порядка $n$ содержит не более $n-1$ квазигрупп. Если она содержит ровно $n-1$ квазигрупп, то ее называют полной системой ортогональных квазигрупп.

\section{§ 2. Построение ОЛК с использованием групп Фробениуса}

Тематика, связанная с ортогональными латинскими квадратами и квазигруппами, является классической, и к настоящему времени имеется весьма обширная научная литература, посвященная этой области, лежащей на стыке комбинаторной математики и алгебры. Большое внимание ОЛК уделено в монографиях [7], [14]. Основные проблемы теории ОЛК заключаются в построении латинских квадратов, ортогональных заданному квадрату, и в построении максимальных, в частности, полных, систем ОЛК. До сих пор остается открытой проблема описания натуральных чисел $n$, для которых существует полная система ОЛК.

Первые серьезные результаты по ОЛК принадлежат Эйлеру. Он в 1782 г. доказал существование пар ОЛК порядка $n$ для всех нечетных $n$ и высказал гипотезу об отсутствии пар ОЛК порядка $n=2(2 k-1)$. В 1921 г. Макнейч [26], построил полную систему ОЛК для любого простого числа $n$ МАТЕМАТИЧЕСКИЕ ВОПРОСЫ КРИПТОГРАФИИ 
и высказал гипотезу о том, что максимальная система ОЛК для любого $n$ на единицу меньше минимального примарного делителя числа $n$. Гипотезы Эйлера (при $n>6$ ) и Макнейча долго оставались открытыми, и обе были опровергнуты в работах Бозе, Шриканда и Паркера [9-11], [31-32]. В итоге были построены полные системы ОЛК любых примарных порядков $p^{k}$.

В последующие годы этими и другими авторами был предложен ряд новых подходов к построению ОЛК. Во многих случаях для построения ОЛК в качестве исходных квадратов использовались таблицы Кэли конечных групп.

Ниже предлагается метод построения ортогональных систем квазигрупп и латинских квадратов, основанный на использовании групп Фробениуса, и проводится его сравнение с некоторыми другими методами.

Приведем необходимые сведения о группах Фробениуса см., например, [19], гл. 4, п. 5, и [20], гл. 5, § 8).

Пусть $G-$ группа подстановок на множестве $Q=\{0,1, \ldots, n-1\}$. Для любых точек $a, b, \ldots, c \in Q$ обозначим через $G_{a, b, \ldots, c}$ подгруппу группы $G$ всех подстановок, оставляющих на месте каждую из точек $a, b, \ldots, c$. Эта подгруппа называется стабилизатором точек $a, b, \ldots, c$ в группе $G$. Для подстановки $g \in G$ точка а $\in Q$ называется стабильной, если $g(a)=a$, и мобильной в противном случае.

Группа $G$ называется группой Фробениуса, если выполнены условия:

1) $G$ транзитивна;

2) $\left|G_{a}\right|>1 \quad \forall a \in Q$

3) $\left|G_{a, b}\right|=1 \forall a, b \in Q, a \neq b$.

Из условия 2 следует, что группа Фробениуса не регулярна, а из условия 3 следует, что для любых двух пар $(a, b),(c, d)$ элементов множества $Q$ при $a \neq b, c \neq d$ в $G$ существует не более одной подстановки, переводящей первую пару во вторую.

Группы Фробениуса достаточно хорошо изучены. Все подстановки без стабильных элементов группы Фробениуса $G$ вместе с единичной подстановкой образуют регулярную характеристическую подгруппу (а потому и нормальный делитель) $R$ группы $G$ порядка $n=|Q|$, и группа $G$ представляется в виде полупрямого произведения $G=R H$ групп $R, H$, которые называются соответственно ядром и дополнительной подгруппой группы $G$. При этом централизатор любого неединичного элемента $r \in R$ в группе $G$ содержится в $R$, а нормализатор группы $H$ в $G$ совпадает с $H$. В качестве $H$ может служить любой стабилизатор $G_{a}$, причем стабилизаторы различных элементов пересекаются лишь по единичной подстановке. По одной из фундаментальных теорем Томпсона группа $R$ нильпотентна. Если $|H|$ четно, то $R$ абелева, a $H$ - группа с единственной инволюцией. Если $|H|$ нечетно, то $H$ метациклическая, т. е. расширение циклической группы с помощью циклической груп- 
пы. В любом случае силовская $p$-подгруппа группы $H$ является либо циклической, либо обобщенной группой кватернионов и только циклической при нечетном $p$.

Из условий 1-3 по известной лемме Бернсайда получаем: $|G|=n k$, где $k=\left|G_{a}\right|-$ делитель числа $n-1$. Как и в любой регулярной группе, каждая подстановка группы $R$ является регулярной, а каждая неединичная подстановка из $G \backslash R$ оставляет неподвижным ровно один элемент из $Q$ и является регулярной на множестве остальных элементов.

Пусть $G-$ группа Фробениуса с ядром $R=\left\{r_{0}, \ldots, r_{n-1}\right\}$, и индексы у подстановок из $R$ выбраны так, что $0 r_{a}=a$. При таком выборе индексов отображение $f: R \rightarrow Q$ по правилу $f\left(r_{a}\right)=a$ будет изоморфизмом группы $R$ на группу $(Q,+) c$ операцией $a+b=a r_{b}$, подстановка $r_{b}-$ правой трансляцией, a $R$ - правым регулярным представлением группы $(Q,+)$. Разложив группу $G$ в объединение правых смежных классов по подгруппе $R$ :

$$
G=R h_{1} \cup R h_{2} \cup \ldots \cup R h_{k},
$$

мы получим $k$ упорядоченных регулярных множеств подстановок

$$
R h_{i}=\left(r_{0} h_{i}, \ldots, r_{n-1} h_{i}\right), i=1,2, \ldots, k,
$$

где $R h_{1}=R$. Записав каждую подстановку из $R h_{i}$ в виде таблицы из двух строк с одной и той же стандартной верхней строкой $0,1, \ldots, n-1$ и записав последовательно одну под другой нижние строки всех подстановок из $R h_{i}$, получим латинский квадрат $L_{i}$. Проделав это для всех $i=1,2, \ldots, k$, получим $k$ латинских квадратов. Добавив еще к этим квадратам стандартные входные строки и столбцы, мы получим $k$ квазигрупп $\left(Q, *_{i}\right), i=1, \ldots, k$. Заметим, что при $i=1$ операция $*_{i}$ совпадает с операцией + в группе $(Q,+)$. Обозначим через $I_{i}$ и $\backslash_{i}$ операции соответственно левого и правого деления в квазигруппе $\left(Q, *_{i}\right)$.

В итоге мы получили три системы квазигрупп $\left(Q, *_{i}\right),\left(Q, l_{i}\right),\left(Q, \backslash_{i}\right)$, $i=1, \ldots, k$.

Аналогичным образом можно получить $k$ упорядоченных регулярных множеств подстановок по разложению группы $G$ в левые смежные классы

$$
h_{i} R=\left(h_{i} r_{0}, \ldots, h_{i} r_{n-1}\right), i=1, \ldots, k,
$$

и по ним построить еще три системы квазигрупп, которые обозначим в виде

$$
\left(Q, \circ_{i}\right),\left(Q, / I_{i}\right),\left(Q, \|_{i}\right), i=1, \ldots, k .
$$

Заметим, что смежные классы $R h_{i}$ и $h_{i} R$ как множества подстановок совпадают, поскольку $R-$ нормальный делитель в $G$. Однако как упорядо- 
ченные множества они в общем случае различны, а потому различны и порождаемые ими серии квазигрупп.

Теорема 1. Если $G=R H-$ группа Фробениуса с ядром $R$ и с дополнительной подгруппой $H$ порядка $k$, то в указанных выше обозначениях каждая из следующих систем квазигрупп является ортогональной:

$$
\text { a) }\left(Q, /_{i}\right), \text { b) }\left(Q, \backslash_{i}\right), \text { c) }\left(Q, \circ_{i}\right), \text { d) }\left(Q, / /_{i}\right), i=1, \ldots, k \text {. }
$$

Доказательство. Не теряя общности, будем считать, что $H=\left\{h_{0}, \ldots, h_{n-1}\right\}=$ $=G_{0}$. Обозначим:

$$
\begin{gathered}
R h_{i}=\left(s_{0}, \ldots, s_{n-1}\right), R h_{j}=\left(t_{0}, \ldots, t_{n-1}\right), s_{a}=r_{a} h_{i}, t_{a}=r_{a} h_{j}, a \in Q, \\
h_{i} R=\left(u_{0}, \ldots, u_{n-1}\right), h_{j} R=\left(v_{0}, \ldots, v_{n-1}\right), u_{a}=h_{i} r_{a}, v_{a}=h_{j} r_{a}, a \in Q .
\end{gathered}
$$

Тогда операции $*_{i}, *_{j}, o_{i}, \circ_{j}$, соответствующие упорядоченным множествам подстановок $R h_{i}, R h_{j}, h_{i} R, h_{j} R$, будут задаваться равенствами

$$
a *_{i} b=b s_{a}=b r_{a} h_{i}, a *_{j} b=b t_{a}=b r_{a} h_{j}, a \circ_{i} b=b u_{a}=b h_{i} r_{a}, a \circ_{j} b=b v_{a}=b h_{j} r_{a} .
$$

Рассмотрим теперь отдельно утверждения a)-d).

a) Допустим, что квазигруппы $\left(Q, /_{i}\right),\left(Q, /_{j}\right)$ не ортогональны, т. е. найдутся такие $a, b \in Q$, при которых система уравнений

$$
\begin{gathered}
x l_{i} y=a, \\
x /_{j} y=b
\end{gathered}
$$

имеет два различных решения $\left(x_{1}, y_{1}\right),\left(x_{2}, y_{2}\right)$. Тогда в соответствии с эквивалентностями (2) имеем равенства

$$
a *_{i} y_{1}=x_{1}, \quad b *_{j} y_{1}=x_{1}, \quad a *_{i} y_{2}=x_{2}, \quad b *_{j} y_{2}=x_{2},
$$

которые в силу (4) можно записать в следующем виде:

$$
y_{1} s_{a}=x_{1}, \quad y_{1} t_{b}=x_{1}, \quad y_{2} s_{a}=x_{2}, \quad y_{2} t_{b}=x_{2} .
$$

Отсюда получаем равенства $y_{1} t_{b} s_{a}{ }^{-1}=y_{1}, y_{2} t_{b} s_{a}{ }^{-1}=y_{2}$, которые по определению группы Фробениуса могут выполняться лишь при $t_{b} s_{a}^{-1}=\varepsilon$, т. е. при $t_{b}=s_{a}$. Последнее равенство противоречиво, поскольку подстановки $t_{b}$ и $s_{a}$ содержатся в разных смежных классах группы $G$ по $R$. Следовательно, квазигруппы $\left(Q, /_{i}\right),\left(Q, /_{j}\right)$ ортогональны.

b) Допустим, что квазигруппы $\left(Q, \backslash_{k}\right),\left(Q, \backslash_{i}\right)$ не ортогональны, т.е. система уравнений

$$
\begin{gathered}
x \backslash_{i} y=a, \\
x \backslash_{j} y=b
\end{gathered}
$$

имеет два различных решения $\left(x_{1}, y_{1}\right),\left(x_{2}, y_{2}\right)$. Тогда в соответствии с эквивалентностями (2) имеем равенства

$$
x_{1} *_{i} a=y_{1}, \quad x_{1} *_{j} b=y_{1}, \quad x_{2} *_{i} a=y_{2}, \quad x_{2} *_{j} b=y_{2},
$$


которые в силу (3) можно записать в следующем виде:

$$
a s_{x_{1}}=y_{1}, \quad b t_{x_{1}}=y_{1}, \quad a s_{x_{2}}=y_{2}, \quad b t_{x_{2}}=y_{2} \text {. }
$$

Отсюда получаем: $a s_{x_{1}}=b t_{x_{1}}, a s_{x_{2}}=b t_{x_{2}}$ и $a s_{x_{1}} t_{x_{1}}^{-1}=a s_{x_{2}} t_{x_{2}}^{-1}$. Следовательно, $a s_{x_{1}} t_{x_{1}}^{-1} t_{x_{2}} s_{x_{2}}^{-1}=a, \quad$ и потому $\quad s_{x_{1}} t_{x_{1}}^{-1} t_{x_{2}} s_{x_{2}}^{-1} \in G_{a}$. Однако $s_{x_{1}} t_{x_{1}}^{-1} t_{x_{2}} s_{x_{2}}^{-1}=$ $r_{x_{1}} h_{i} h_{j}^{-1} r_{x_{1}}^{-1} r_{x_{2}} h_{j} h_{i}^{-1} r_{x_{2}}^{-1}=r_{x_{1}} h r h^{-1} r_{x_{2}}^{-1}$, где $h=h_{i} h_{j}^{-1} \in G_{0}, r=r_{x_{1}}^{-1} r_{x_{2}} \in R$. Отсюда, учитывая, что $R-$ нормальный делитель группы $G$, получаем $s_{x_{1}} t_{x_{1}}^{-1} t_{x_{2}} s_{x_{2}}^{-1} \in R$. А так как $R \cap G_{a}=\{\varepsilon\}$, то $s_{x_{1}} t_{x_{1}}^{-1} t_{x_{2}} s_{x_{2}}^{-1}=\varepsilon$, и, следовательно,

$$
r_{x_{1}} h r_{x_{1}}^{-1} r_{x_{2}} h^{-1} r_{x_{2}}^{-1}=\varepsilon \text {, т.е. } r_{x_{1}} h r_{x_{1}}^{-1}=r_{x_{2}} h r_{x_{2}}^{-1} \text {. }
$$

Так как $h \in G_{0}$, то $r_{x_{1}} h r_{x_{1}}^{-1} \in G_{x_{1}}, r_{x_{2}} h r_{x_{2}}^{-1} \in G_{x_{2}}$. Однако в группе Фробениуса $G_{x_{1}} \cap G_{x_{2}}=\{\varepsilon\}$ при $x_{1} \neq x_{2}$, и потому равенство $r_{x_{1}} h r_{x_{1}}^{-1}=r_{x_{2}} h r_{x_{2}}^{-1}$ возможно лишь при $x_{1}=x_{2}$. Тогда, очевидно, и $y_{1}=y_{2}$, и мы приходим к противоречию.

c) Допустим, что квазигруппы $\left(Q, \circ_{i}\right),\left(Q, \circ_{j}\right)$ не ортогональны, т. е. система уравнений

$$
\begin{gathered}
x \circ_{i} y=a, \\
x \circ_{j} y=b
\end{gathered}
$$

имеет два различных решения $\left(x_{1}, y_{1}\right),\left(x_{2}, y_{2}\right)$. Тогда из (3) получаем равенства

$$
y_{1} h_{i} r_{x_{1}}=a, \quad y_{1} h_{j} r_{x_{1}}=b, \quad y_{2} h_{i} r_{x_{2}}=a, \quad y_{2} h_{j} r_{x_{2}}=b .
$$

Из них нетрудно получить соотношение

$$
a r_{x_{1}}^{-1} h r_{x_{1}} r_{x_{2}}^{-1} h^{-1} r_{x_{2}}=a \text {, где } h=h_{i}^{-1} h_{j} .
$$

Далее, как и в п. b, доказывается равенство $r_{x_{1}}^{-1} h r_{x_{1}} r_{x_{2}}^{-1} h^{-1} r_{x_{2}}=\varepsilon$, ведущее к противоречию.

d) В этом случае доказательство проводится по аналогии с доказательством утверждения а). Теорема доказана.

Заметим, что системы квазигрупп $\left(Q, *_{i}\right),\left(Q, \backslash_{i}\right), i=1, \ldots, k$, в общем случае не ортогональны.

Таким образом, имея группу Фробениуса $G=R H$, мы сможем легко построить 4 системы попарно ортогональных квазигрупп (латинских квадратов) порядка $n=|R|$, каждая из которых содержит $k=|H|$ квазигрупп (латинских квадратов).

В связи с построением системы ОЛК порядка $n$ на основании теоремы 1 естественно возникает практически важная проблема построения групп Фробениуса. Для решения последнего вопроса необходимо выяснить сначала, какие группы могут служить в качестве ядер групп Фробениуса, а затем для каждой такой группы найти дополнительную подгруппу. 
По определению группы Фробениуса ее ядро должно быть регулярной группой подстановок. Такую группу можно получить в качестве левого регулярного представления любой конечной группы. Однако далеко не все регулярные группы подстановок могут служить ядрами групп Фробениуса. По известной теореме Томпсона ядрами групп Фробениуса могут служить лишь некоторые нильпотентные группы и даже абелевы, если $|H|$ четно. В последнем случае $H$ содержит единственную инволюцию, и значит, ее силовская 2-подгруппа является либо циклической, либо обобщенной группой кватернионов.

Если $G=R H-$ группа Фробениуса с ядром $R$ и с дополнительной подгруппой $H$, то каждый элемент $h \in H$ индуцирует автоморфизм $\sigma_{h}$ группы $R$, определяемый формулой

$$
\forall r \in R: \sigma_{h}(r)=h^{-1} r h .
$$

Этот автоморфизм при любом $h$ является регулярным. Таким образом, ядром группы Фробениуса может быть лишь регулярная группа подстановок, допускающая нетривиальный регулярный автоморфизм. Ясно, что этот класс групп совпадает с множеством регулярных групп, допускающих регулярный автоморфизм простого порядка. Все конечные абелевы группы с этим свойством описаны автором в работе [4]. Так как силовские подгруппы являются характеристическими, то указанная задача сводится к описанию лишь примарных групп. Результат описания таких групп выделим из работы [4] в качестве отдельной теоремы.

Теорема 2. Конечная абелева р-группа допускает регулярный автоморфизм простого порядка $q$ в том и только том случае, когда в ее разложении в прямую сумму циклических групп число сомножителей $n_{i}$ одного u того же порядка $p^{i}$ удовлетворяет условию: $p^{n_{i}}-1$ кратно $q$.

Описания нильпотентных неабелевых групп, допускающих регулярный автоморфизм простого порядка, автору неизвестны.

Рассмотрим теперь задачу отыскания дополнительной подгруппы $H$ для группы Фробениуса $G$ с фиксированным ядром $R$.

Теорема 3. Пусть $R$ - левое регулярное представление абелевой группь $(Q,+)$. Тогда дополнительными подгруппами подстановок с неподвижным нулем групп Фробениуса с ядром $R$ являются нетривиальные группы регулярных автоморфизмов группы $(Q,+)$ и только они.

Доказательство. Пусть $G=R H$ - группа Фробениуса $c$ ядром $R$ и $H=G_{0}$. Докажем, что $H$ есть группа регулярных автоморфизмов группы $(Q,+)$. Выясним, как связаны элементы из $H$ с регулярными автоморфизмами группы $(Q,+)$. Так как группы $R$ и $(Q,+)$ изоморфны, то любой автомор- 
физм $\varphi$ группы $(Q,+)$ индуцирует автоморфизм $\varphi^{\prime}$ группы $R$, который определяется формулой

$$
\varphi^{\prime}\left(r_{a}\right)=r_{a} \varphi .
$$

И обратно, любой автоморфизм группы $R$ индуцируется указанным выше образом автоморфизмом группы $(Q,+)$. Иначе говоря, формулой (5) устанавливается взаимно однозначное соответствие между автоморфизмами групп $R$ и $(Q,+)$. Очевидно, что при этом регулярным автоморфизмам соответствуют регулярные автоморфизмы.

Выше было отмечено, что любой элемент $h \in H$ индуцирует регулярный автоморфизм $\sigma_{h}$ группы $R$. В силу отмеченной связи между автоморфизмами групп $R$ и $(Q,+)$ имеем: $\sigma_{h}=\varphi^{\prime}$ для некоторого автоморфизма $\varphi$ группы $(Q,+)$. Следовательно, элемент $h$ удовлетворяет системе подстановочных уравнений Коши:

$$
h^{-1} r_{a} h=\varphi^{\prime}\left(r_{a}\right), a \in Q .
$$

Так как подстановки $r_{a}$ и $\varphi^{\prime}\left(r_{a}\right)$ являются регулярными и одного и того же порядка, то они имеют одинаковую цикловую структуру. Следовательно, каждое из уравнений системы (6) имеет решение. А так как группа $R$ транзитивна, то система (6) имеет не более одного решения $h$ с условием $h(0)=0$. Легко проверить, что системе уравнений (6) удовлетворяет автоморфизм $\varphi^{\prime}$ (как подстановка множества $Q$ ). Действительно, используя обозначение $x \varphi=y$ и равенство (5), получим:

$$
y+r_{a} \varphi=(x \varphi) \varphi^{-1} r_{a} \varphi=x r_{a} \varphi=(a+x) \varphi=a \varphi+x \varphi=a \varphi+y=y r_{a} \varphi=y \varphi^{\prime}\left(r_{a}\right) .
$$

Следовательно, $h=\varphi$ и группа $H$ есть группа регулярных автоморфизмов группы $(Q,+)$.

Обратно, пусть $R=\left\{r_{0}, \ldots, r_{n-1}\right\}-$ регулярная группа подстановок множества $Q$, где, как и выше, $0 r_{a}=a$, и $H-$ некоторая нетривиальная группа регулярных автоморфизмов группы $(Q,+)$. Докажем, что $G=R H-$ группа Фробениуса с ядром $R$ и с дополнительной подгруппой $H=G_{0}$. Из условия $0 r_{a}=a$ следует, что $x r_{a}=0 r_{x} r_{a}=0 r_{a+x}=a+x$, и потому для любого автоморфизма $\varphi \in H$ :

$$
x r_{b} \varphi=(b+x) \varphi=b \varphi+x \varphi=x \varphi r_{b} \varphi .
$$

Отсюда следует, что $R \varphi=\varphi R$, а значит, и $R H=H R$. Следовательно, $G-$ группа и $R$ - ее нормальный делитель. Очевидно, что для группы $G$ условия 1-2 из определения группы Фробениуса выполнены, остается проверить 
условие 3. Пусть для некоторых $\varphi \in \mathrm{H}, a, x, y \in Q$ выполнены равенства $x r_{a} \varphi=x, y r_{a} \varphi=y$. Тогда

$$
(a+x) \varphi=x, \quad(a+y) \varphi=y, \quad \text { т. е. } a \varphi+x \varphi=x, \quad a \varphi+y \varphi=y .
$$

Отсюда следует, что $-(a \varphi+y \varphi)+(a \varphi+x \varphi)=-y+x$, т. е. $-y \varphi-a \varphi+a \varphi+$ $+x \varphi=-y+x$, т. е. $(-y+x) \varphi=-y+x$. Так как автоморфизм $\phi$ регулярный, то последнее равенство возможно лишь при $x=y$. Следовательно, условие 3 выполнено и $\mathrm{G}$ - группа Фробениуса. Теорема доказана.

Приведем в качестве следствий из теорем 1-3 два примера на построение систем ОЛК с использованием регулярных автоморфизмов конечных абелевых групп,

Следствие 1. Для любого челого числа $n=p^{k}$, где $p-$ простое число $u k>0$, существует полная система ортогональных латинских квадратов порядка п.

Для доказательства укажем путь построения искомой системы ОЛК. Пусть $(Q,+)$ - элементарная абелева $p$-группа порядка $p^{k}$ и $R-$ ее правое регулярное представление. На ней естественным образом задается $k$-мерное линейное пространство над полем $\mathrm{GF}(p)$. При этом автоморфизмами группы $(Q,+)$ будут линейные преобразования пространства. Известно, что линейное преобразование с примитивным характеристическим многочленом имеет порядок $p^{k}-1$ и, значит, порождает группу $H$ регулярных автоморфизмов порядка $p^{k}-1$. Тогда $R H-$ группа Фробениуса, по которой и строится полная система ОЛК по алгоритму, указанному в доказательстве теоремы 1.

Заметим, что других групп Фробениуса порядка $n(n-1)$ с ядром порядка $n$ не существует, т. е. с помощью групп Фробениуса полные системы ОЛК непримарных порядков построить нельзя.

Следствие 2. Пусть $n=p_{1}^{m_{1}} \ldots p_{k}^{m_{k}}-$ каноническое разложение натурального числа п. Тогда существует система ОЛК порядка $n$, состоящая из $N=$ НОД $\left\{p_{i}^{m_{i}}-1: i=1, \ldots, k\right\}$ квадратов.

Для доказательства возьмем в качестве группы $(Q,+)$ прямую сумму элементарных абелевых $p_{i}$-групп и для каждой из них найдем регулярный автоморфизм порядка $N$ как подходящую степень автоморфизма максимального порядка, построенного в следствии 1. Построенные таким образом автоморфизмы прямых слагаемых будут индуцировать требуемый регулярный автоморфизм порядка $N$ группы $(Q,+)$.

Выясним еще, как выражаются операции $*_{i}, l_{i}, \iota_{i},{ }_{i}, / l_{i}$ через операции группы $(Q,+)$ и подстановку $h_{i} \in H \subseteq \operatorname{Aut}(Q,+)$. Согласно (3) имеем:

$$
a *_{i} b=b s_{a}=b r_{a} h_{i}=(a+b) h_{i}=a h_{i}+b h_{i} .
$$


Отсюда в соответствии с правилом (2) из соотношения $a f_{i} b=c$ получаем $c *_{i} b=a=c h_{i}+b h_{i}$. Следовательно, $c=\left(a-b h_{i}\right) h_{i}^{-1}=a h_{i}^{-1}-b$. Таким образом,

$$
a l_{i} b=a h_{i}^{-1}-b .
$$

Аналогично получим: $a \backslash_{i} b=-a+b h_{i}^{-1}, a \circ_{i} b=a+b h_{i}, a / l_{i} b=a-b h_{i}$.

Отсюда видно, что все квазигруппы из систем (4) главно изотопны группе $(Q,+)$, причем компоненты изотопий являются автоморфизмами группы $(Q,+)$. В частности, все эти квазигруппы линейны. Латинские квадраты, являющиеся их таблицами Кэли, получаются из таблицы Кэли группы $(Q,+)$ с помощью перестановок строк и столбцов.

Рассмотрим еще вопрос о построении систем ортогональных квазигрупп, изотопных неабелевым группам. Как было отмечено выше, ядро группы Фробениуса является нильпотентной группой. Напомним (см., например, [7]) что группа $(G, \cdot)$ называется нильпотентной ступени $c$, если для нее существует нормальный ряд подгрупп

$$
E=Z_{0}<Z_{1}<\ldots<Z_{c}=G,
$$

в котором $E-$ единичная группа, $Z_{1}-$ центр группы $G, Z_{i+1} / Z_{i}-$ центр группы $G / Z_{i}, i=0, \ldots, c-1$. Наличие такого ряда эквивалентно существованию другого нормального ряда

$$
E=G_{0}<G_{1}<\ldots<G_{c}=G
$$

где $G_{c-1}$ - коммутант группы $G, G_{i}$ - взаимный коммутант групп $G_{i+1}$ и $G$. Ряды (7), (8) называются соответственно верхним и нижним центральными рядами группы $G$.

В качестве примера нильпотентной неабелевой группы чаще всего приводят группу $\mathrm{UT}(n, P)$ всех унитреугольных матриц размеров $n \times n$ над полем $P$ при $n>2$ (см., например, [5], [19]). Она является нильпотентной ступени $n-1$. В ней ряды (7), (8) совпадают, а подгруппа $G_{i}$ из ряда (8) является подгруппой матриц $\mathrm{c} n-i-1$ нулевыми диагоналями над главной диагональю.

Так как нас интересуют лишь конечные группы, то будем рассматривать группу $G=\mathrm{UT}(n, P)$ при любом $n>2$ и $P=\mathrm{GF}\left(p^{m}\right)$, где $p-$ любое простое число. Для построения группы Фробениуса с ядром $R$, изоморфным группе $G$, необходимо найти группу регулярных автоморфизмов группы $G$. Приведем класс таких групп, указанных Н. Ито (см. [20], стр. 499).

Выберем простой делитель $q \geq n$ числа $p^{m}-1$, попарно различные элементы $d_{1}, \ldots, d_{n}$ поля $P$, удовлетворяющие условию $d_{i}^{q}=1, i=1, \ldots, n$, и такое число $k$, что $0<k<q$ и $(k, q)=1$. Теперь определим автоморфизм $\psi: G \rightarrow G$, положив для любого $g \in G: \psi(g)=h^{-k} g h^{k}$, где $h=\operatorname{diag}\left(d_{1}, \ldots, d_{n}\right)$. Непосредственной проверкой легко убедиться, что $\psi$ - регулярный автомор- 
физм порядка $q$ группы $G$. В итоге мы получим группу Фробениуса порядка $p^{\frac{n(n-1)}{2}} q$ и сможем построить систему из $q$ ОЛК порядка $p^{\frac{n(n-1)}{2}}$. В частности, при $p=2$ в качестве $2^{m}-1$ можно выбрать число Мерсенна, и тогда мы сможем получить систему из $2^{m}-1$ ОЛК порядка $2^{\frac{n(n-1)}{2}}$ при любом $n>2$.

\section{§ 3. О методе Стивенса-Боуза построения полной системы ОЛК}

Первые работы о построении полных систем ОЛК были опубликованы Боузом в 1938 г. (см. [9]) и Стивенсом в 1939 г. (см. [33]). Их интерес к этой теметике был связан с замеченными к тому времени связями между ОЛК и проективными и аффинными плоскостями. А именно, было установлено, что проективная (а потому и аффинная) плоскость порядка $n$ существует тогда и только тогда, когда существует полная система ОЛК порядка $n$. Имеются достаточно простые алгоритмы построения плоскостей по полным системам ОЛК и обратно. Описание этих алгоритмов можно найти, например, в книге В. Н. Сачкова [6]. Там же приводится и метод Стивенса-Боуза построения полной системы ОЛК. Он для $n=p^{m}$, где $p-$ простое число, заключается в следующем.

Берется поле $P=\mathrm{GF}\left(p^{m}\right)=\left\{a_{0}=0, a_{1}=1, a_{2}, \ldots, a_{n-1}\right\}$ и над ним определяется система из $n-1$ матриц $A_{r}=\left(a_{i, j}^{(r)}\right), r=1, \ldots, n$, размеров $n \times n$ с элементами

$$
a_{i, j}^{(r)}=a_{i} a_{r}+a_{j} .
$$

Доказывается, что полученные таким образом матрицы образуют полную систему ОЛК.

Нас интересует вопрос о связи этого метода с методом построения ОЛК на основе групп Фробениуса. Добавив к полученным матрицам входные строки и столбцы с указанным выше расположением элементов поля $P$, мы получим систему из $n-1$ квазигрупп $\left(P, \bullet_{r}\right)$, где $a \bullet_{r} b=a a_{r}+b$.

Нетрудно заметить, что полученная система ОЛК сопряжена с системой квазигрупп $\left(Q, \circ_{i}\right), i=1, \ldots, k$, из теоремы 1 при условии, что в качестве группы $(Q,+)$ взята аддитивная группа поля $P$, а в качестве ее группы регулярных автоморфизмов - группа отображений $H=\left\{h_{r}: r=1, \ldots, n-1\right.$, $\left.h_{r}(x)=x a_{r}\right\}$.

Действительно, при этих условиях имеем: если $R-$ регулярное представление группы $(P,+)$, то $R H-$ группа Фробениуса и $a \circ_{r} b=b h_{r} r_{a}=a+b a_{r}$. Отсюда и видно, что операции $\bullet{ }_{r}$ и ${ }_{r}$ сопряжены. 


\section{§ 4. О построении ортогональных квазигрупп с использованием ортоморфизмов групп}

В 1942 г. Манном в работе [27] для построения ортогональных квазигрупп было введено и использовалось понятие полной подстановки группы.

Подстановка $h$ группы $(Q,+)$ называется полной, если отображение $f: Q \rightarrow Q$, определенное формулой $f(x)=x+h(x)$, является подстановкой. Группа, имеющая полную подстановку, называется допустимой. Оказалось, что для группы $(Q, \cdot)$ ортогональная квазигруппа существует тогда и только тогда, когда существует полная подстановка группы $(Q,+)$. При этом в указанных выше обозначениях ортогональной к $(Q,+)$ будет квазигруппа $(Q, \circ)$, где

$$
x \circ y=-x+f(x+y) \text {. }
$$

В работах Л. Пейджа [29] и М. Холла [30] было найдено необходимое и достаточное условие существования полной подстановки группы. Оказалось, что допустимыми являются все конечные группы, кроме групп четного порядка с циклической силовской 2-подгруппой. В частности, допустимыми являются все симметрические группы подстановок $S_{n}$ при $n>3$.

В работе [29] для получения ОЛК было введено близкое к полной подстановке понятие ортоморфизма группы. Подстановка $h$ группы $(Q,+)$ (не обязательно коммутативной) называется ее ортоморфизмом, если отображение $f: Q \rightarrow Q$, определенное формулой $f(x)=x-h(x)$, является подстановкой множества $Q$. Таким образом, подстановка $x \rightarrow h(x)$ группы является ортоморфизмом тогда и только тогда, когда подстановка $x \rightarrow-h(x)$ является полной. Легко видеть, что все регулярные (в частности, правильные) автоморфизмы группы являются ее ортоморфизмами. Интересно отметить, как авторы по ортоморфизму $h$ группы $(Q,+)$ строят ортогональную ей квазигруппу. Сначала они составляют таблицу Кэли группы в так называемом стандартном виде, когда элементы во входной строке и входном столбце располагаются так, что нулевые элементы внутри таблицы располагаются на главной диагонали. Вторую же таблицу получают из первой путем перестановки строк по подстановке $h$. Нетрудно видеть, что если входные строки и столбцы построенных таблиц записать при одном и том же расположении элементов, то первая таблица превратится в таблицу вычитания, т. е. будет таблицей квазигруппы $(Q, /)$, вторая же получается из первой точно так же, как таблицы $\left(Q, /_{i}\right)$ при использовании группы Фробениуса, где в качестве $h$ выступали автоморфизмы группы $(Q,+)$. Таким образом, метод ортоморфизмов можно рассматривать как обобщение метода, основанного на группах Фробениуса. 
Доказывается, что квазигруппа $Q_{h}$ ортогональна группе $(Q,+)$ тогда и только тогда, когда $h-$ ортоморфизм группы $(Q, \cdot)$. Две квазигруппы $Q_{h}$ и $Q_{g}$ ортогональны тогда и только тогда, когда ортоморфизмом является отображение $h^{-1} g$. Тем самым построение систем квазигрупп, ортогональных к заданной группе, сводится к построению системы ортоморфизмов этой группы. Однако общие простые методы решения последней задачи не известны. Один из имеющихся методов основан на связи ортоморфизмов группы с трансверсалями ее таблицы Кэли.

Диагональю квадрата $\left(a_{i, j}\right)$ порядка $n$ называется множество, состоящее из $n$ пар $(i, j)$, в которых все первые, а также и все вторые компоненты попарно различны. Иначе говоря, это множество мест квадрата, взятых по одному из каждой его строки и каждого столбца. Трансверсалью латинского квадрата $\left(a_{i, j}\right)$ порядка $n$ на множестве $Q$ и соответствующей ему квазигруппы называется множество элементов из $Q$ с указанием номеров их мест, расположенных на любой одной его диагонали, при условии, что в нем каждый элемент из $Q$ встречается ровно один раз.

Отсюда следует, что наличие одной трансверсали группы влечет существование разбиения ее таблицы Кэли в объединение попарно непересекающихся трансверсалей. Таким образом, для группы существует ортогональная ей квазигруппа тогда и только тогда, когда в ее таблице Кэли существует хотя бы одна трансверсаль. В общем случае это не так, и потому представляет определенный интерес задача определения числа трансверсалей у заданного латинского квадрата и максимума $T(n)$ этой величины по всем квадратам заданного порядка $n$. Оценки для $T(n)$ можно найти, например, в [23]. Краткий обзор работ по трансверсалям можно найти в [34].

В работе [21] для групп небольших порядков (от 3 до 12) поиск ортоморфизмов группы производится с помощью компьютерных вычислений. Используя ортоморфизмы групп, авторы находят максимальные системы ОЛК небольших порядков. Приведем полученные ими результаты.

Для $n=3,4,5,6$ все ортоморфизмы групп (если они существуют) являются автоморфизмами, и значит, все ортогональные им квазигруппы можно построить с помощью групп Фробениуса.

Для $n=7$ существует 20 ортоморфизмов, из которых 6 - автоморфизмы. Полная система ортогональных квазигрупп получается лишь с использованием автоморфизмов, т. е. может быть построена с помощью группы Фробениуса.

Для $n=8$ циклическая группа не имеет ортоморфизмов, группа $\mathbf{Z} / 4+\mathbf{Z} / 2$ не имеет правильных нетривиальных автоморфизмов, но имеется 49 ортоморфизмов, по которым строятся 24 ортогональные системы по 3 квадрата. Это первый пример максимальных ортогональных систем, которые не могут быть построены с помощью групп Фробениуса. В наиболее интересном слу- 
чае, когда группа является прямой суммой трех групп порядка 2, все ортоморфизмы являются правильными автоморфизмами и полная система ОЛК может быть построена с помощью групп Фробениуса.

Для $n=9$ циклическая группа имеет 226 ортоморфизмов, максимальная ортогональная система состоит из двух квадратов, которые могут быть построены с помощью групп Фробениуса. Если же группа является прямой суммой двух подгрупп порядка 3 , то ортоморфизмами являются лишь автоморфизмы, и некоторые полные системы ОЛК могут быть построены с помощью групп Фробениуса.

Для $n=10$ существует лишь циклическая группа, и она не имеет ортоморфизмов. Для $n=11$, как и для $n=7$, строятся 3432 ортоморфизма, не являющихся автоморфизмами, и полная система ОЛК может быть построена с помощью групп Фробениуса.

Для $n=12$ циклическая группа не имеет ортоморфизмов, для группы $\mathbf{Z} / 6+\mathbf{Z} / 2$ найдена максимальная система ОЛК порядка 12: она состоит из 5 квадратов, что больше известной ранее оценки $\sqrt{n}$. Отметим, что правильных автоморфизмов эта группа не имеет, и потому это есть пример, когда максимальная система ОЛК, получаемая из абелевой группы, не может быть получена с использованием групп Фробениуса.

\section{§ 5. О построении ортогональных квазигрупп с использованием операции Манна на множестве латинских квадратов фиксированного порядка}

Еще один подход к построению систем ОЛК основан на введенной Манном в [27] бинарной операции на группоидах с заданным основным множеством $Q$. Для группоидов с операциями $f, g$ Манн определил группоид с операцией $h=f \cdot g$ на $Q$, положив

$$
h(x, y)=f(x, g(x, y)) \text {. }
$$

В случае, когда исходные группоиды являются квазигруппами, операция Манна приводит к следующей операции умножения латинских квадратов. Пусть $L, R$ - латинские квадраты в алфавите $Q$ и $\left\{g_{1}, \ldots, g_{n}\right\},\left\{h_{1}, \ldots, h_{n}\right\}-$ соответствующие им регулярные множества подстановок, упорядоченные по возрастанию номеров соответствующих строк квадратов $L, R$.

Произведением латинских квадратов $L, R$ называется таблица, составленная из нижних строк подстановок $g_{1} h_{1}, \ldots, g_{n} h_{n}$. Эта операция естественным образом индуцирует операцию умножения соответствующих квазигрупп. В общем случае введенное произведение латинских квадратов (квазигрупп) может не быть латинским квадратом (квазигруппой). В статьях [15-17] рассматривается вопрос о получении ОЛК с помощью степеней латинских квадратов. Легко видеть, что если для латинского квадрата $L$ квадМАТЕМАТИЧЕСКИЕ ВОПРОСЫ КРИПТОГРАФИИ 
раты $L^{2}, \ldots, L^{m}$ также являются латинскими, то все $m$ квадратов обязательно попарно ортогональны. Если при этом число $m$ выбрано наибольшим, то множество $\left\{L, L^{2}, \ldots, L^{m}\right\}$ называют степенным множеством латинских квадратов (Latin power set). Сопоставив латинским квадратам $L, L^{2}, \ldots, L^{m}$ соответствующие им квазигруппы, получим степенное множество квазигрупп (quasigroup power set). В [15] высказана гипотеза о существовании степенного множества из $m \geq 2$ латинских квадратов порядка $n$ для любого $n \neq 2,6$ и доказано, что эта гипотеза верна для всех $n$ при $0 \leq n \leq 50$ и всех $n$, бо́льших 50, исключая, возможно, числа вида $6 k+2$. Их метод был основан на известной схеме Мендельсона [21], которая дает бесконечно много контпримеров к гипотезе Эйлера, но, к сожалению, не применима при $n \equiv 2$ (mod 6). В работе [17] предложен новый способ конструирования степенных множеств латинских квадратов (квазигрупп) порядка $p$ для любого простого $p \geq 11$. (Из них уже можно получать степенные множества квазигрупп и латинских квадратов составных размеров известным способом, используя прямые разложения алгебр.) Их метод основан на использовании различных упорядочиваний подстановок заданной регулярной группы подстановок. Однако, как отмечают сами авторы, для способа шифрования, предложенного в [18], предпочтительнее иметь метод построения степенных множеств квазигрупп, не основанный на группах, поскольку большая иррегулярность делает шифр более надежным.

В связи с этим в работе [8] предлагаются 2 более общих способа построения степенных множеств. Первый основан на использовании уравновешенных неполных блок-схем индекса 1 (BIB $(v, b, r, k, 1))$, в которых множество $Q$ из $v$ элементов разбито на $b$ блоков $Q_{1}, \ldots, Q_{b}$ порядка $k$ так, что каждый элемент входит ровно в $r$ блоков и каждая пара различных элементов входит ровно в один блок. Второй - на использовании дважды уравновешенных блок-схем индекса 1 и типа $\left(v ; k_{1}, \ldots, k_{m}\right)$, в которых множество из $v$ элементов разбито на $b$ блоков, из которых $b_{i}$ блоков содержат по $k_{i}$ элементов, $i=1, \ldots, s$, и так, что каждая пара различных элементов входит ровно в один блок.

Суть обоих методов заключается в том, что сначала для случая, когда $k$ есть степень простого числа, строится степенное множество квазигрупп с операциями $A, A^{2}, \ldots, A^{k-2}$ на конечном поле $Q=\mathrm{GF}(k)$, определяемыми по формулам

$$
A^{i}(x, y)=\left(1-a^{i}\right) x+a^{i} y, i=1, \ldots, k-2,
$$

где $a-$ примитивный элемент поля $H, 1-$ единица поля $H$.

Теорема 4. Квазигруппь $\left(Q, A^{i}\right), i=1, \ldots, k-2$, могут быть получены с помощью групп Фробениуса. 
Доказательство. Обозначим через $R_{i}$ множество правых трансляций квазигруппы $\left(Q, A^{i}\right)$ и через $R_{i, y}$ - правую трансляцию, соответствующую элементу $y$. Напомним, что

$$
R_{i, y}(x)=A^{i}(x, y)=\left(1-a^{i}\right) x+a^{i} y, i \in\{1, \ldots, k-2\}, y \in \mathrm{GF}(k) .
$$

Добавим еще к указанным квазигруппам группу $(Q,+)$ - аддитивную группу поля $\mathrm{GF}(k)$, обозначив группу ее трансляций через $R_{0}=\left\{R_{0, y}: y \in \mathrm{GF}(k)\right\}$. Теперь докажем, что $G=\bigcup_{i=0}^{k-2} R_{i}-$ группа Фробениуса с ядром $R_{0}$. Для этого докажем сначала, что $G$ - группа, т. е. $G$ замкнуто относительно операции умножения подстановок. Пусть $x, y, z \in Q$. Тогда

$$
\begin{gathered}
x R_{i, y} R_{j, z}=\left(\left(1-a^{i}\right) x+a^{i} y\right) R_{j, z}=\left(1-a^{j}\right)\left(\left(1-a^{i}\right) x+a^{i} y\right)+a^{j} z= \\
=\left(1-a^{j}\right)\left(\left(1-a^{i}\right) x+\left(1-a^{j}\right) a^{i} y+a^{j} z .\right.
\end{gathered}
$$

Заметим, что уравнение $\left(1-a^{j}\right)\left(\left(1-a^{i}\right)=\left(1-a^{k}\right)\right.$ разрешимо относительно $k$. Действительно, из него находим $a^{k}=a^{i}+a^{j} a^{i+j}$, и если элемент $a^{i}+a^{j}-a^{i+j} \neq 0$, то он является некоторой степенью примитивного элемента $a$. Тогда показатель степени и даст искомое $k$. Допустим, что $a^{i}+a^{j}-a^{i+j}=0$. Тогда при $i \geq j$ получим равенство $1+a^{i-j}-a^{i}=0$, что противоречит тому, что $a$ - примитивный элемент поля. Аналогично придем к противоречию и при $i<j$. Таким образом, имеем $R_{i, y} R_{j, z}=R_{k, u}$, где $u$ однозначно находится из соотношения $\left(1-a^{j}\right) a^{i} y+a^{j} z=a^{k} u$. Следовательно, $G-$ группа подстановок на множестве $Q$. Она транзитивна, так как транзитивна ее подгруппа $R_{0}$, и не регулярна, так как $|Q|>1$. Очевидно, что неединичные подстановки из $R_{0}$ не имеют неподвижных точек. Пусть $x$ - неподвижная точка подстановки $R_{i, y}$, где $i \neq 0$. Тогда $\left(1-a^{i}\right) x+a^{i} y=x$ и $x=y$. Таким образом, каждая подстановка из $G \backslash R_{0}$ оставляет неподвижной ровно одну точку. В итоге получаем: $G-$ группа Фробениуса с ядром $R_{0}$. При этом множества $R_{i}$ являются правыми смежными классами группы $G$ по подгруппе $R_{0}$. Действительно, при любых фиксированных $z, y$ :

$$
\begin{gathered}
x R_{0, z} R_{i, y}=(x+z) R_{i, y}=\left(1-a^{i}\right)(x+z)+a^{i} y=\left(1-a^{i}\right) x+\left(1-a^{i}\right) z+ \\
+a^{i} y=\left(1-a^{i}\right) x+a^{i} u=R_{i, u},
\end{gathered}
$$

где $u$ однозначно находится из условия $\left(1-a^{i}\right) z+a^{i} y=a^{i} u$. Следовательно, $R_{0} R_{i}=R_{i}$, т. е. $R_{i}-$ смежный класс.

Обозначим через $\backslash_{i}$ операцию на $Q$, правую обратную к $A^{i}$. Тогда $x \backslash_{i}$ $y=z \Leftrightarrow A^{i}(x, z)=y \Leftrightarrow\left(1-a^{i}\right) x+a^{i} z$ и, следовательно, $x \backslash i \quad y=\left(1-a^{-i}\right) x+$ $+a^{-i} y$. Отсюда видно, что построенная ОЛК совпадает с системой ОЛК из п. $\mathrm{b}$ теоремы 1 , что и требовалось доказать.

\section{Список литературы}

1. Белоусов В. Д. Основы теории квазигрупп и луп. - М.: Наука, 1967.

2. Белоусов В. Д. Алгебраические сети и квазигруппы. - Кишинев, Штиинца, 1971. 
3. Белоусов В. Д. Системы ортогональных операций. - Матем. сб., 1968, т. 77 (119), № 1, с. 38-58.

4. Глухов М. М., Ларин С. В. Абелевы и метабелевы группы с регулярными автоморфизмами и полуавтоморфизмами. - Матем. заметки, 1972, т. 12, вып. 6, с. 727-738.

5. Каргополов М. И., Мерзляков Ю. И. Основы теории групп. - М.: Наука, 1972.

6. Сачков В. Н. Комбинаторные методы дискретной математики. - М.: Наука, 1977.

7. Холл М. Комбинаторика. - М.: Мир, 1970.

8. Belyavskaya G. B. Quasigroup power sets and cyclic $S$-systems. - Quasigroups and related Systems, 2002, v. 9, p. 1-17.

9. Bose R. C. On application of the properties of Galois fields to the problem of construction of hyper-Graeco-Latin squares. - Sankhya, 1938, v. 3, p. 323338.

10. Bose R. C., Nair K. R. On complete sets of orthogonal Latin squares. Sankhya, 1941, p. 361-382.

11. Bose R. C., Shrikhande S. S., Parker E. T. Further results on the constructions of mutually orthogonal Latin squares and the falsity Euler's conjecture. Canad. J. Math., 1960, v. 12, p. 189-203.

12. Brack R. H. Finite nets, I. — Canad. J. Math., 1951, v. 3, p. 94-107.

13. Brack R. H. A survey of binary systems. - Springer Verlag, BerlinHeidelberg-Gottingen, 1958.

14. Denes J., Keedwell A. D. Latin Squares. New Developments in the Theory and Applications. - Nord-Holland Publishing Co., Amsterdam, 1981.

15. Denes J., Mullen G. L., Suchower S. J. A note on power sets of Latin squares. - J. Combin. Computing, 1994, v. 16, p. 27-31.

16. Denes J. When is there a Latin power set? - Amer. Math. Monthly, 1997, v. 104, p. 563-565.

17. Denes J., Owens P. J. Some new power sets not based on groups. - J. Combin. Theory, Ser. A, 1999 , v. 85 , p. 69-82.

18. Denes J., Petroczki P. A digital encrypting communication systems. - Hungarian Patent № 201437A, 1990.

19. Gorenstein D. Finite groups. - Harper and Row, New York, Evanston, London, 1968.

20. Huppert B. Endliche Gruppen, I. - Springer Verlag, Berlin-Heidelberg-New York, 1967.

21. Johnson D. M., Dulmage A. L., Mendelson N. S. Orthomorphisms of groups and orthogon Latin squares, I. — Canad. J. Math., 1961, v. 13, p. 356-372.

22. Keedwell A.D. On R-sequenceability and $\mathrm{R}_{\mathrm{h}}$-sequenceability of groups. Combinatorics `81, North-Holland Math / Stud., 1983, v. 78, p. 535-548. 
23. Koksma K. K. Lower bound for the order of a partial transversal in a Latin square. - J. Combinatorial Theory, 1969, v. 7, p. 94-95.

24. Laywine C. F., Mullen G. L. Discrete mathematics using Latin squares. John Wiley\&Sons, Inc., New York, 1998.

25. Leakh I. V. On transformations of orthogonal systems of optrations and algebraic nets. - Ph. D. thesis, IM AN RM, Kishinev, 1986.

26. MacNeish H. F. Euler squares. - Ann. Math., 1921, v. 23, p. 221-227.

27. Mann H. B. The construction orthogonal Latin squares. - Ann. Math. Statist., 1942 , v. 13 , p. $418-423$.

28. Norton D. A. Group of orthogonal row-latin squares. - Pacific J. Math., 1952, v. 2, p. 335-341.

29. Paige L. J. Complete mappings of finite groups. - Pacific J. Math., 1951, v. 1, p. 111-116.

30. Paige L. J., M. Hall. Complete mappings of finite groups. Pacific J. Math., 1955, v. 5, p. 541-549.

31. Parker E. T. Construction of some sets of pairwise orthogonal Latin squares. - Proc. Amer. Math. Soc., 1959, v. 10, p. 949-951.

32. Parker E. T. Orthogonal Latin squares. - Proc. Natural Academ. Sci. U.S.A., 1959 , v. 45 , p. 859-962.

33. Stevens $W$. The Completely orthogonalized Latin squares. - Ann.Eugen, 1939, v. 9, p. 269-307.

34. Wanless I. M. Transversals in Latin squares. - Quasigroups and related systems, 2007, v. 15, № 1, p. 169-190. 